\title{
Local finite-time Lyapunov exponent, local sampling and probabilistic source and destination regions
}

\author{
A. E. BozorgMagham ${ }^{1}$, S. D. Ross ${ }^{2}$, and D. G. Schmale III ${ }^{3}$ \\ ${ }^{1}$ Department of Atmospheric and Oceanic Science, University of Maryland, College Park, MD 20742, USA \\ ${ }^{2}$ Department of Biomedical Engineering and Mechanics, Virginia Tech, Blacksburg, VA 24061, USA \\ ${ }^{3}$ Department of Plant Pathology, Physiology, and Weed Science, Virginia Tech, Blacksburg, VA 24061, USA
}

Correspondence to: A. E. BozorgMagham (bozorgmagham@gmail.com)

Received: 28 April 2015 - Published in Nonlin. Processes Geophys. Discuss.: 28 May 2015

Revised: 2 September 2015 - Accepted: 19 October 2015 - Published: 11 November 2015

\begin{abstract}
The finite-time Lyapunov exponent (FTLE) is a powerful Lagrangian concept widely used for describing large-scale flow patterns and transport phenomena. However, field experiments usually have modest scales. Therefore, it is necessary to bridge the gap between the concept of FTLE and field experiments. In this paper, two independent observations are discussed: (i) approximation of the local FTLE time series at a fixed location as a function of known distances between the destination (or source) points of released (or collected) particles and local velocity, and (ii) estimation of the distances between the destination (or source) points of the released (or collected) particles when consecutive release (or sampling) events are performed at a fixed location. These two observations lay the groundwork for an ansatz methodology that can practically assist in field experiments where consecutive samples are collected at a fixed location, and it is desirable to attribute source locations to the collected particles, and also in planning of optimal local sampling of passive particles for maximal diversity monitoring of atmospheric assemblages of microorganisms. In addition to deterministic flows, the more realistic case of unresolved turbulence and low-resolution flow data that yield probabilistic source (or destination) regions are studied. It is shown that, similar to deterministic flows, Lagrangian coherent structures (LCS) and local FTLE can describe the separation of probabilistic source (or destination) regions corresponding to consecutively collected (or released) particles.
\end{abstract}

\section{Introduction}

The classical interpretation of finite-time Lyapunov exponent (FTLE) fields and the associated hyperbolic Lagrangian coherent structures (LCSs) provides useful information about large-scale flow patterns and transport and mixing phenomena in flow domains (Haller and Poje, 1998; Haller and Yuan, 2000; Mancho et al., 2004; Shadden et al., 2005; Haller, 2011). There are an increasing number of studies that apply various concepts of LCSs, based on the classic right CauchyGreen tensor, to describe and predict the time evolution of Lagrangian features in geophysical systems (Haller, 2015). In some of these studies, geophysical information (e.g., wind or oceanic velocity fields) have been used as the input data, and Lagrangian results (e.g., the distribution of an oil spill in the ocean or volcanic ash in the atmosphere) over a large area are compared with the behavior of the geophysical system via satellite data or simulations (Dellnitz et al., 2009; Peng and Peterson, 2012; Olascoaga and Haller, 2012; Mendoza and Mancho, 2012; Olascoaga et al., 2012). A large-scale distribution of particles is a common characteristic among these studies. In contrast, this study is motivated by a series of field experiments regarding the long-distance transport of airborne microorganisms where only a limited number of localized and temporally consecutive measurements of the atmospheric structure of microbial assemblages are available (Schmale III et al., 2008; Tallapragada et al., 2011; Schmale et al., 2012; Lin et al., 2013; Schmale and Ross, 2015). Therefore, there is a need to bridge the powerful concept of FTLE and local field experiments. 


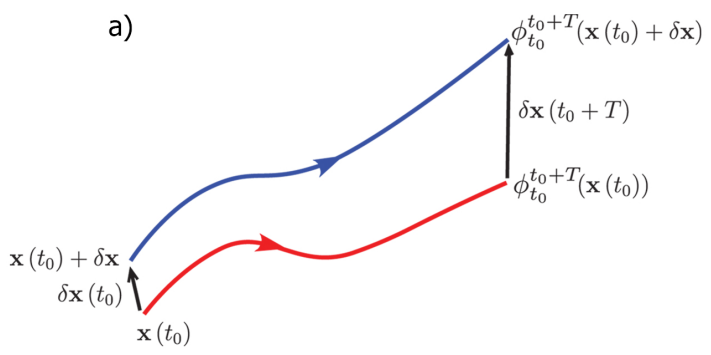

b)

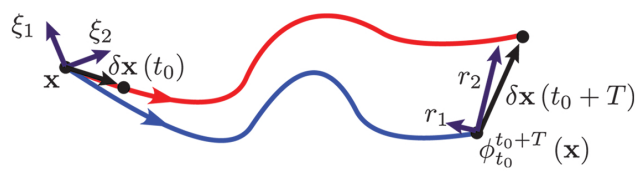

Figure 1. (a) Separation of nearby particles during time interval $T$ due to the flow map $\phi$. The two particles are released in the flow field at the same time $t_{0}$; isochronic particles. (b) $\boldsymbol{\xi}_{2}$ is the direction of maximum growth at the initial point $\boldsymbol{x}$, which evolves into the direction $\boldsymbol{r}_{2}=D \phi_{t_{0}}^{t_{0}+T}(\boldsymbol{x}) \boldsymbol{\xi}_{2}$ at the evolved point $\phi_{t_{0}}^{t_{0}+T}(x)$. The closer the initial displacement vector $\delta x$ is to the $\boldsymbol{\xi}_{2}$ direction, the more it will be stretched to that maximum perturbation.

In this paper, we present two independent observations related to the estimation of the local FTLE and the distance between destination (or source) points of released (or collected) particles. These observations provide an ansatz for bridging field experiment results with the concept of local Lyapunov exponents and the direction of maximum expansion in ordinary differential equation systems; however, a rigorous mathematical formalism for non-autonomous dynamical systems is still needed (Abarbanel et al., 1992; Branicki and Wiggins, 2009; Kloeden and Rasmussen, 2011). These observations may help investigate long-distance transport phenomena as a possible cause of variation in successively collected airborne samples such as the presence or absence of a unique strain or species of microorganism. In addition, this analysis is useful for planning geophysical sampling at a fixed location with respect to forecast FTLE fields (BozorgMagham et al., 2013).

Because this study is motivated by aerial measurements in realistic conditions, i.e., hundreds of collections of microorganisms from the atmosphere with drones, it is necessary to consider the spatiotemporal limitations of the available velocity field data. These limitations are manifested in unresolved turbulence and impose uncertainties on the locations of the source and destination points. For this reason, we use a Lagrangian particle dispersion model to determine the probabilistic source (or destination) regions and show how the concept of a local FTLE and deterministic Lagrangian coherent structures (LCS) can explain the separation between probabilistic source (or destination) regions, and may contribute to understanding the geographic and genetic diversity observed in aerial samples (Fay et al., 1995; Draxler and Hess, 1998; BozorgMagham and Ross, 2015).

Results from this study can be applied to environmental applications such as early warning systems for airborne pathogens, integrated pest management in crops, and the collection of samplers from geophysical flows (Tallapragada et al., 2011; BozorgMagham et al., 2013; BozorgMagham and Ross, 2015).

This paper is outlined as follows. In Sect. 2 we present two observations associated with the estimation of the local FTLE and the dispersion of destination (or source) points in flow fields. In Sect. 3 we show some numerical examples and applications of presented observations in periodic and aperiodic systems. In Sect. 4 we consider the unresolved turbulence and investigate the uncertainty of the backward and forward trajectories and the resulting probabilistic source and destination regions.

\section{Local finite-time Lyapunov exponent}

In this section we present two independent observations related to the estimation of the local FTLE and the distance between destination (or source) points of successively released (or collected) particles in a time-varying $n$-dimensional vector field,

$\frac{\mathrm{d} \boldsymbol{x}}{\mathrm{d} t}=\boldsymbol{v}(\boldsymbol{x}, t)$,

where $n=2$ for two-dimensional flows and $n=3$ for threedimensional flows.

By local FTLE we mean the time-varying value of the FTLE field at an arbitrary location $\boldsymbol{x}$. Classically, the timevarying FTLE measures the maximum separation rate between nearby particles when they are released in the flow field at the same time (isochronic particles). Figure 1a refers to this classical description. This figure shows two isochrone particles that are close to each other at an initial time $t_{0}$. Under the effect of the flow field, the small displacement vector between the two particles, $\delta \boldsymbol{x}$, changes. After an elapsed time $T$, the new vector between the two particles is

$$
\begin{aligned}
\delta \boldsymbol{x}\left(t_{0}+T\right) & =\phi_{t_{0}}^{t_{0}+T}(\boldsymbol{x}+\delta \boldsymbol{x})-\phi_{t_{0}}^{t_{0}+T}(\boldsymbol{x}) \\
& =D \phi_{t_{0}}^{t_{0}+T}(\boldsymbol{x})+O\left(\left|\delta \boldsymbol{x}\left(t_{0}\right)\right|^{2}\right),
\end{aligned}
$$

where $\phi_{t_{0}}^{t_{0}+T}$ is the flow map for the vector field (Eq. 1) from time $t_{0}$ to $t_{0}+T, D \phi_{t_{0}}^{t_{0}+T}=\mathrm{d} \phi_{t_{0}}^{t_{0}+T}(\boldsymbol{x}) / \mathrm{d} \boldsymbol{x}$ is the Jacobian of the flow map, and $|\cdot|$ is the Euclidean norm.

Consider the right Cauchy-Green strain tensor, $C\left(\boldsymbol{x}, t_{0}\right.$, $T)=D \phi_{t_{0}}^{t_{0}+T}(\boldsymbol{x})^{\top} D \phi_{t_{0}}^{t_{0}+T}(\boldsymbol{x})$. For the sake of the follow- 

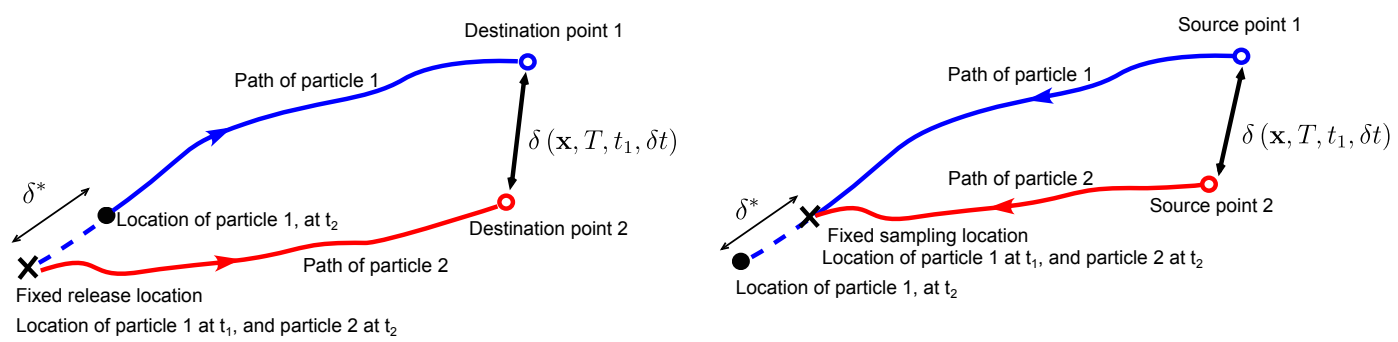

Figure 2. Two sequentially released (left panel)/collected (right panel) particles at a fixed location shown by a bold cross. Particles 1 and 2 are released/collected at $t_{1}$ and $t_{2}=t_{1}+\delta t$, respectively (the time interval between the two sequential samplings is $\delta t$ ). The integration time between the destination/sources and the release/sampling location is approximately $T$ for both particles $(|T| \gg \delta t)$. The displacement of the first particle during $\delta t$ is shown by $\delta^{*}$.

ing discussion, consider the situation of incompressible twodimensional flows, $n=2$. The eigenvalues $\lambda_{i}$ and normalized eigenvectors $\xi_{i}$ of $C$ satisfy (Haller, 2015)

$$
\begin{aligned}
C \xi_{i}= & \lambda_{i} \xi_{i}, \quad\left|\xi_{i}\right|=1, \quad i=1,2, \quad 0<\lambda_{1}<1<\lambda_{2}, \\
& \xi_{1} \perp \xi_{2},
\end{aligned}
$$

where the $\left(\boldsymbol{x}, t_{0}, T\right)$ dependence of $C, \lambda_{i}$, and $\xi_{i}$ is understood. As illustrated in Fig. 1b, the two eigenvectors, $\xi_{1}$ and $\xi_{2}$, are carried along by the flow $\phi_{t_{0}}^{t_{0}+T}$ to the two vectors $\boldsymbol{r}_{1}$ and $\boldsymbol{r}_{2}$, respectively, where

$\boldsymbol{r}_{i}=D \phi_{t_{0}}^{t_{0}+T}(\boldsymbol{x}) \boldsymbol{\xi}_{i}$

whose lengths are scaled by a factor $\sqrt{\lambda_{i}}$ compared with the normalized eigenvectors. The maximum possible separation between the released particles after a time interval $T$, assuming a sufficiently small initial distance $\left|\delta \boldsymbol{x}\left(t_{0}\right)\right|$, is

$\max \left|\delta \boldsymbol{x}\left(t_{0}+T\right)\right|=\sqrt{\lambda_{\max }\left(C\left(\boldsymbol{x}, t_{0}, T\right)\right)}\left|\delta \boldsymbol{x}\left(t_{0}\right)\right|$

where $\lambda_{\max }=\lambda_{n}$.

The finite-time Lyapunov exponent (FTLE), with $t_{0}$ and $T$ fixed, is considered a scalar field of the Lyapunov exponent as a function of initial position, $\boldsymbol{x}$,

$\sigma_{t_{0}}^{T}(\boldsymbol{x})=\frac{1}{|T|} \ln \sqrt{\lambda_{\max }(C)}$.

Similar to the calculation of maximum separation between two initially neighboring points in a system of ordinary differential equations (ODEs) and the corresponding maximum Lyapunov exponents, $\sigma_{t_{0}}^{T}$ can be used, via Eqs. (5) and (6), to describe $\max \left|\delta \boldsymbol{x}\left(t_{0}+T\right)\right|$ as

$\max \left|\delta \boldsymbol{x}\left(t_{0}+T\right)\right|=\exp \left(\sigma_{t_{0}}^{T}\left(\boldsymbol{x}, t_{0}\right)|T|\right)\left|\delta \boldsymbol{x}\left(t_{0}\right)\right|$.

In this study we are interested in particles that are released (or collected) sequentially in time at a fixed location. Thus, the standard concept of the FTLE, i.e., the separation rate of nearby isochronic points, might not be applicable. Therefore, we present two independent observations and show that we can (i) approximate the local FTLE by using the information of local velocity and successive destination (or source) points, and (ii) estimate the distance between the destination (or source) points by having the local FTLE and velocity. These two observations require the assumption of a timedependent vector field, so that the initial displacement vector is not along a common trajectory for sequential particles.

Referring to Fig. 2, left panel, assume that two particles are sequentially released at $t_{1}$ and $t_{2}=t_{1}+\delta t$ at the release location shown by the $\mathbf{x}$. The right panel corresponds to the analogous situation of sequentially collected particles.

Observation I: the local FTLE value over the time interval $\left[t_{1}, t_{2}\right]$, given an appropriate $0<\delta t \ll|T|$, can be approximated by

$\sigma_{\left[t_{1}, t_{2}\right]}^{T}(\boldsymbol{x})=\frac{1}{|T|} \ln \frac{\delta\left(\boldsymbol{x}, T, t_{1}, \delta t\right)}{\left|\overline{\boldsymbol{v}}\left(\boldsymbol{x}, t_{1}, t_{2}\right) \delta t\right|}$,

where $\delta\left(\boldsymbol{x}, T, t_{1}, \delta t\right)$ is the distance between successive destination (or source) points corresponding to the elapsed time $T$, and $\overline{\boldsymbol{v}}\left(\boldsymbol{x}, t_{1}, t_{2}\right)$ is the (non-zero) average velocity at the release (or sampling) location during $\left[t_{1}, t_{2}\right]$.

Observation II: the distance between the destination (or source) points of consecutively released (or collected) particles can be estimated, given an appropriate $\delta t$, by the local velocity and the true local FTLE at the release (or sampling) location as

$\delta\left(\boldsymbol{x}, T, t_{1}, \delta t\right)=\exp \left(|T| \sigma_{\left(t_{1}, t_{2}\right)}^{T}(\boldsymbol{x})\right)\left|\overline{\boldsymbol{v}}\left(\boldsymbol{x}, t_{1}, t_{2}\right) \delta t\right|$.

We suggest that observation I provides a recovered FTLE field, based on mild assumptions that tend to hold in geophysical flows. Observation II is important for sampling purposes, because it enables us to estimate the distance between the source positions of consecutively collected particles, if we have the local velocity and local FTLE data by separate means. We note that observations I and II are independent, and the information on the right-hand sides of Eqs. (8) and (9) is also assumed known. For example, the local velocity could be obtained from an anemometer or high-frequency radar in the ocean (Shadden et al., 2009), or the local FTLE could be obtained from a nowcast or forecast velocity field. 


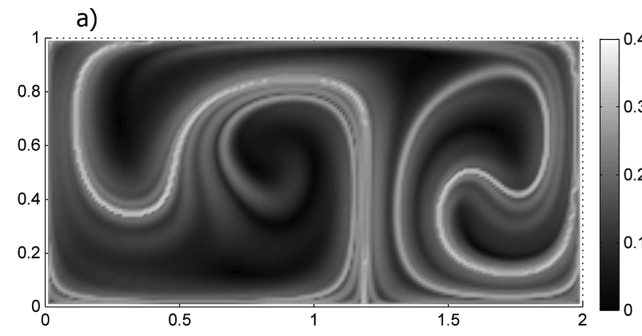

b)

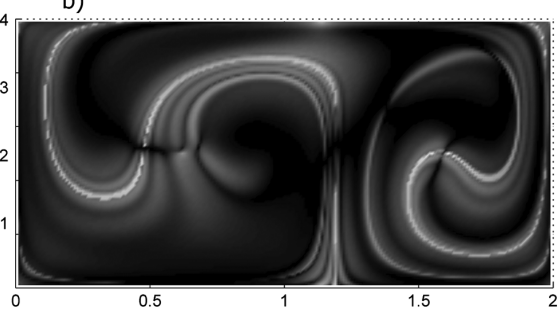

Figure 3. Forward FTLE field of a periodic double-gyre velocity field. (a) The benchmark FTLE field at $t_{0}=1$ calculated by Eq. (6). (b) The approximated FTLE field calculated by Eq. (8), and $\delta t=0.2$ time units. A fourth-order Runge-Kutta integration scheme with constant integration time step 0.01 and total integration time $T=15$ time units is implemented for both panels.

Two remarks are in order regarding these observations. (i) A proper choice of $\delta t$, which depends on the spatiotemporal variability of the velocity field, is critical for a good approximation of the local FTLE or the distance between the destination (or source) points. If $\delta t$ is chosen to be too small, then variation of the velocity field would not be observed, the two particles would essentially be following one another along a nearly identical trajectory, and consequently the separation between the two particles would lead to a null LE value, not maximal growth. However, if $\delta t$ is too large, then the initial particle separation at time $t_{2}$ is too large to justify the linearization assumption underlying FTLE calculations; see, e.g., Eq. (2). Thus, a good selection of $\delta t$ depends on the spatiotemporal variability of the velocity field. (ii) A larger true local FTLE of the real flow field yields a smaller error of estimations for the recovered local FTLE and the distance between the destination (or source) points. This comes from numerical evidence in the following sections.

The fundamental idea behind these observations is related to more general methods of analysis of chaotic dynamical systems, often used in experimental settings, namely, that the direction of maximum expansion dominates the dynamics of typical displacement vector growth (see Fig. 1b) (Oseledec, 1968; Abarbanel et al., 1992; Rosenstein et al., 1993; Tanaka and Ross, 2009). This notion is generally accepted in settings assumed to be modeled by underlying autonomous ODEs (whether known or unknown), but to our knowledge, there is no similar theorem for non-autonomous ODEs. Our observations show that in a time-dependent velocity field, with a proper choice of $\delta t$ and sufficiently large $|T|, \delta\left(\boldsymbol{x}, T, t_{1}, \delta t\right)$ is often close to the maximum possible distance between the two particles. These observations, at the present stage, are more of an ansatz, and may help stimulate rigorous mathematical investigation related to separation of non-isochronic nearby particles in a non-autonomous ODE setting.

In the following section we demonstrate some numerical verification and applications of these observations.

\section{Numerical examples and applications}

\subsection{Numerical examples of observations I and II, for periodic and aperiodic velocity fields}

First, we study the well-known example of a periodic doublegyre. We consider the same model and parameters introduced in Sect. 6, Example 1, of Shadden et al. (2005). For observation $\mathrm{I}$, we need to know $\delta\left(\boldsymbol{x}, T, t_{1}, \delta t\right)$ and the local velocity. Therefore, we use the double-gyre model to generate the velocity field and then exploit that data to calculate the trajectories and the corresponding distance between successively released particles after integration time $T$. We repeat this procedure for all the grid points of the gyre domain $[0,2] \times[0,1]$.

Figure $3 \mathrm{a}$ shows the benchmark (true) forward FTLE field corresponding to $t_{0}=1$, calculated by Eq. (6), and Fig. 3b shows the approximated forward FTLE field calculated by Eq. (8). A fourth-order Runge-Kutta integration scheme with constant integration time step 0.01 and total integration time $T=15$ time units is implemented for both panels. For the recovered (approximated) FTLE field, Fig. 3b, we consider $\delta t=0.2$ time units in Eq. (8). One can adjust parameters, e.g., $T$ or $\delta t$, to investigate their impact on the FTLE field.

To investigate observation I for an aperiodic system, we use a two-dimensional model of the time-dependent Rayleigh-Bénard convection model developed by Solomon and Gollub (1988) and implemented by Lekien and Haller (2008) to study unsteady flow separation on slip boundaries. The streamfunction of this model is a function of position and a stochastic time-dependent forcing term.

$\psi(x, y, t)=\frac{A}{k} \sin \{k[x-g(t)]\} \sin (2 y)$

Following Lekien and Haller (2008), we generate the stochastic forcing, $g(t)$, based on a random Fourier spectrum with zero mean and unit impulse covariance (see Fig. 5 in Lekien and Haller, 2008). Figure 4a shows the benchmark forward FTLE field calculated by Eq. (6), and Fig. 4b shows the recovered forward FTLE field calculated by Eq. (8) in the domain $[0,2] \times[0, \pi / 2]$. Two panels of this figure correspond to $t_{0}=1$. Similar to the previous example, a fourth- 


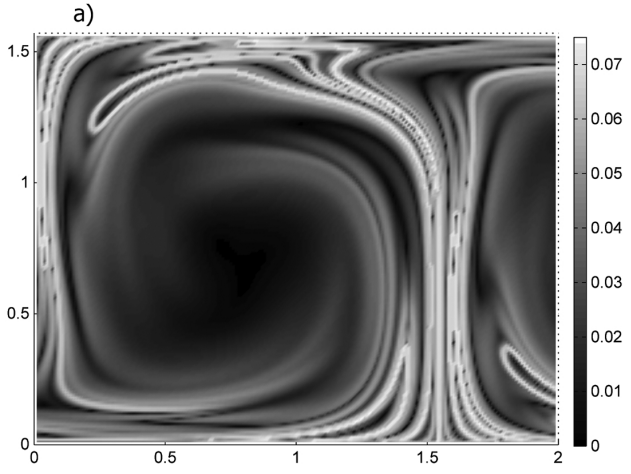

b)

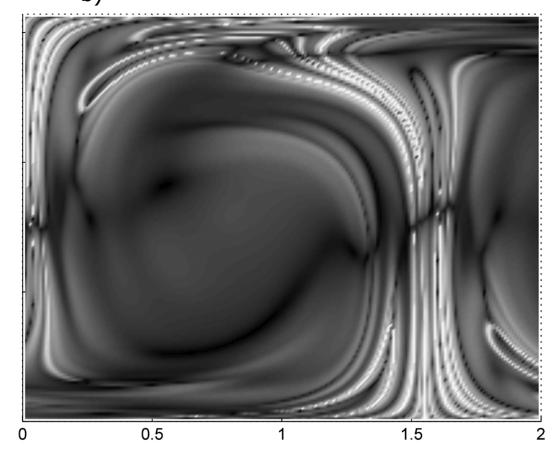

Figure 4. Forward FTLE field of an aperiodic Rayleigh-Bénard convection model. (a) The benchmark FTLE field at $t_{0}=1$ calculated by Eq. (6). (b) The approximated FTLE field calculated by Eq. (8), and $\delta t=0.1$ time units. A fourth-order Runge-Kutta integration scheme with constant integration time step 0.01 and total integration time $T=75$ units is implemented for both panels.
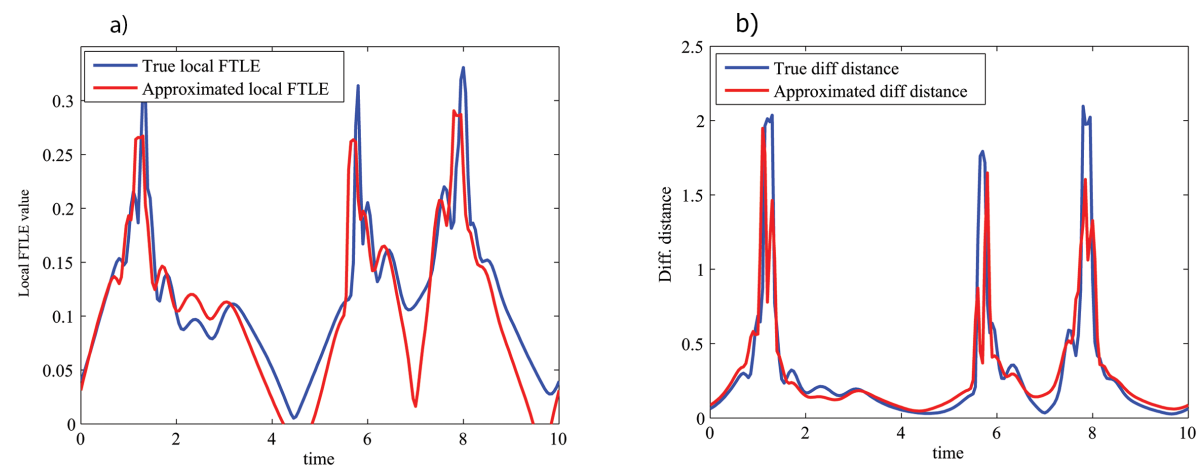

Figure 5. Observations I and II at $(x, y)=(0.3,0.4)$ for a periodic double-gyre velocity field. (a) Local benchmark and recovered forward FTLE. (b) Benchmark and approximated final distance between successively released particles corresponding to $T=15$.

order Runge-Kutta integration scheme with constant integration time step 0.01 is used. The total integration time is $T=75$ time units for both panels. For the recovered FTLE field, Fig. $4 \mathrm{~b}$, we consider $\delta t=0.1$ time units in Eq. (8).

By comparing the two panels of Figs. 3 and 4, respectively, one sees that the main features of the FTLE field are recovered by Eq. (8), and the benchmark and approximated fields are highly correlated. However, in some areas (e.g., near $(1.2,0.5)$ in Fig. 3 and $(1.5,0.8)$ in Fig. 4), we see discontinuities in the recovered FTLE field. The reason might be that the selected $\delta t$ is not a proper choice in those regions. It is also important to note that we use a common color scale for the two panels of Figs. 3 and 4, respectively. Therefore, minute differences between the true and approximated fields are visually exaggerated because the FTLE values are generally small in magnitude. Numerical comparison of the results (see the next two numerical experiments) shows close approximation of the recovered local FTLE to the true values.

Next, we investigate both observations I and II at an arbitrary point over the time span $[0,10]$, which is one period of the double-gyre flow shown in Fig. 3. First, we consider the point $(x, y)=(0.3,0.4)$ in the periodic double-gyre, keeping all the parameters of FTLE computation the same as before (e.g., $\delta t=0.2$ ). Figure 5a shows the benchmark and approximated FTLE time series at that point. The benchmark FTLE is calculated by Eq. (6) using the velocity field information and the maximum eigenvalue of the Cauchy-Green strain tensor. The approximated FTLE in this panel is calculated by Eq. (8). Information about $\delta$ and the local velocity (i.e., $\overline{\boldsymbol{v}}$ ) are assumed to be known (in this numerical experiment we obtain them from the velocity field). Figure 5 b shows the benchmark and approximated distance between successively released particles after an elapsed time $T=15$. To calculate the benchmark time series, we use the velocity field information to generate the trajectories and find the distance between the successive particles. The approximated time series is generated by Eq. (9), with the provided information about the local velocity and the local FTLE value.

Next, we consider the point $(x, y)=(1.3,1.3)$ in the aperiodic time-dependent Rayleigh-Bénard convection model, keeping all the parameters of FTLE computation the same as before (e.g., $\delta t=0.1$ ). Figure 6 a shows the benchmark and approximated FTLE time series at the selected point and 
a)

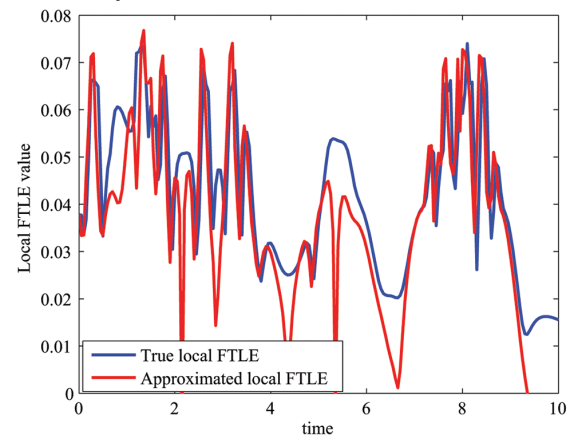

b)

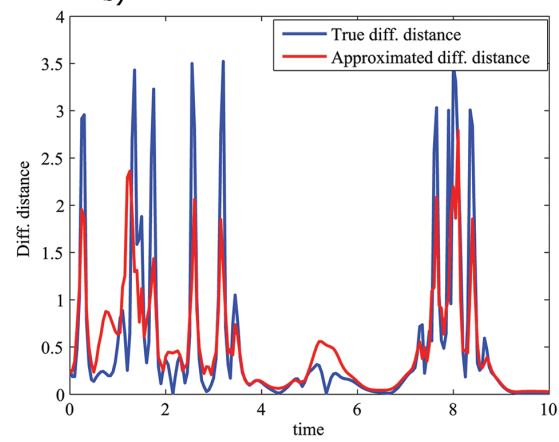

Figure 6. Observations I and II at $(x, y)=(1.3,1.3)$ for an aperiodic velocity field corresponding to a time-dependent Rayleigh-Bénard convection model. (a) Local benchmark and recovered forward FTLE. (b) Benchmark and approximated final distance between successively released particles corresponding to $T=75$.

Fig. $6 \mathrm{~b}$ shows the benchmark and the approximated distance between successively released particles after an elapsed time $T=75$.

Figures 5 and 6 show typical time series of the recovered local FTLE and the distance between successively released (or collected) particles. As one can observe, the two time series in panels $\mathrm{a}$ and $\mathrm{b}$ are highly correlated, and the error of approximation is generally small.

The error of approximation in observations I and II depends on many parameters, for example, $\delta t, T$, and variation of the vector field over the timescale $\delta t$. We leave the analysis of errors of observations I and II for a future study.

\subsection{Applications of the local FTLE observations I and II}

Next, we consider the real-world wind data and focus on the backward FTLE fields and the location of source points. This situation is important for field studies for identifying potential source regions of plant pathogens and their relative risk of transport to previously unexposed regions (Lin et al., 2014; Prussin et al., 2014a, b, 2015). For this purpose, we use observations I and II to compare the benchmark and the recovered local backward FTLE time series and also the true and the estimated distance of the source locations corresponding to the particles that were collected at Virginia Tech's Kentland Farm, located at $37^{\circ} 11^{\prime} \mathrm{N}$ and $80^{\circ} 35^{\prime} \mathrm{W}$. A large variety of microbial samples have been collected at this location over the past 7 years (2006 to 2013) (Schmale et al., 2012). We refer to this point as $(0,0)$ in our plots.

The flow maps are calculated by using numerical data corresponding to the North America Mesoscale, NAM-218, provided by the National Oceanic and Atmospheric Administration (NOAA) and National Centers for Environmental Prediction's (NCEP) Operational Model Archive and Distribution System (NOMADS) project ${ }^{1}$. The spatial resolution of this data set is about $12.1 \mathrm{~km}$ and the temporal resolution

\footnotetext{
${ }^{1}$ http://nomads.ncdc.noaa.gov/data.php
}

is $3 \mathrm{~h}$. All the trajectories are calculated by a fourth-order Runge-Kutta integrator with a constant integration time step equal to $5 \mathrm{~min}$. We use third-order splines for all necessary spatiotemporal interpolations. We consider the time interval 12:00 UTC 29 September to 12:00 UTC 30 September 2010 for our numerical experiments and refer to it as the interrogation window.

Figure 7 shows the trajectories and the initial positions of the indexed particles corresponding to the collected particles at the sampling location during the interrogation window. The frequency of sampling was $1 \mathrm{~h}$ and the backward time integration is $24 \mathrm{~h}$ for all the particles. In addition, for simplicity and without losing generality of the results, we perform the integration on a quasi-two-dimensional $850 \mathrm{mb}$ pressure surface (BozorgMagham and Ross, 2015). Indices of this figure indicate the sampling times of the collected particles; for example, index " 12 " that is located in the northwest of the figure refers to the initial position of a particle that started at 12:00 UTC 28 September and was collected $24 \mathrm{~h}$ later, i.e., 12:00 UTC 29 September, at the sampling location. In terms of streaklines (Batchelor, 2000), this line (see Fig. 7b) is composed of contemporaneous points, e.g., $24 \mathrm{~h}$, from the assembly of streaklines that pass through the sampling location during the interrogation window. We define this line as the isochrone source line since the integration time from all points on it to the sampling location is equal, e.g., $24 \mathrm{~h}$ in this example.

Following the assumptions of the local FTLE observations, i.e., a proper $\delta t$ with respect to the spatiotemporal variability of the velocity field, we choose sampling periods from 0.1 to $1 \mathrm{~h}$, and all the integration is done in the same interrogation window. Figure $8 \mathrm{a}$ shows the benchmark distance between successive source points, i.e., $\delta\left(\boldsymbol{x}, T, t_{1}, t_{2}\right)$, during the interrogation window calculated from the available velocity field data. We use the average velocity at the sampling location to calculate $\delta^{*}$ as $\left|\overline{\boldsymbol{v}}\left(\boldsymbol{x}, t_{1}, t_{2}\right) \delta t\right|$. Figure $8 \mathrm{~b}$ shows the recovered local FTLE time series, assuming that the true successive distances are available. 
a)

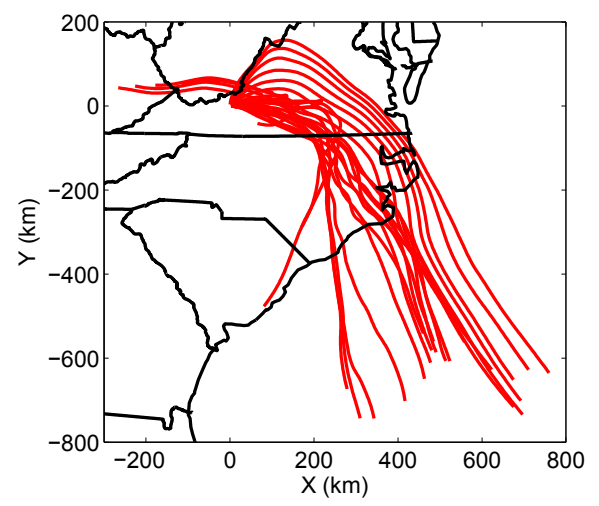

b)

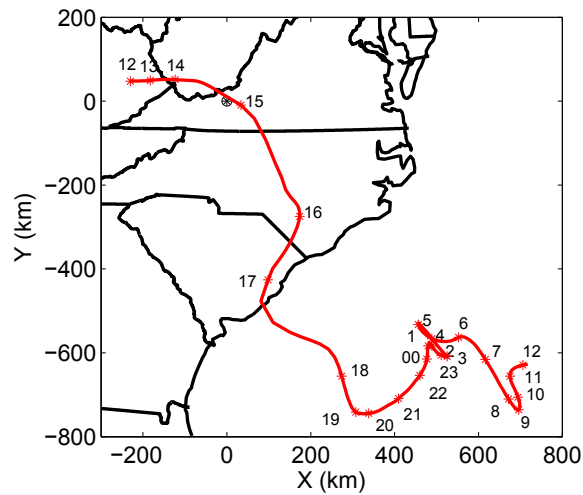

Figure 7. (a) Trajectories of the collected particles during $24 \mathrm{~h}$ of integration. (b) Sequential source points and the isochrone source line. Sampling frequency is $1 \mathrm{~h}$ between 12:00 UTC 29 September and 12:00 UTC 30 September 2010, and the sampling location is at (0, 0) (Virginia Tech Kentland Farm, $37^{\circ} 11^{\prime} \mathrm{N}$ and $80^{\circ} 35^{\prime} \mathrm{W}$ ).

a)

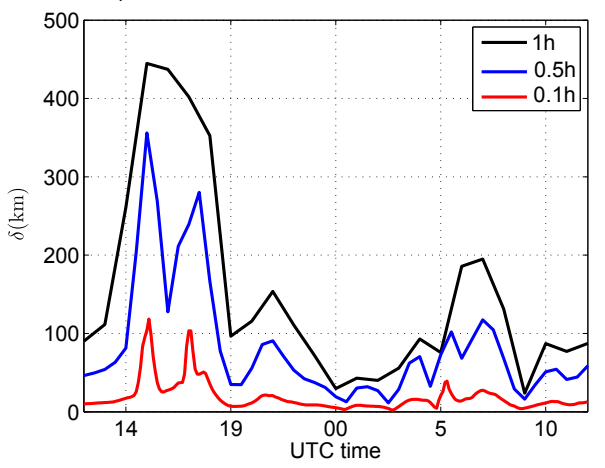

b)

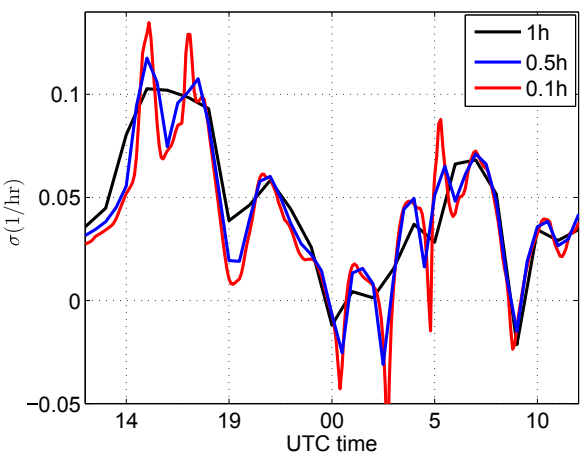

Figure 8. (a) $\delta$ as the benchmark (true) distance between successive source points corresponding to $\delta t=0.1,0.5$ and $1 \mathrm{~h}$. The horizontal axis represents the averaged time corresponding to each successive pair. (b) Approximated local FTLE for different $\delta t \mathrm{~s} \mathrm{from} 0.1 \mathrm{~h}(6 \mathrm{~min})$ to $1 \mathrm{~h}$. The interrogation window is 12:00 UTC 29 September to 12:00 UTC 30 September 2010.

Figures $7 \mathrm{~b}$ and 8 demonstrate that we interpret a local (backward) FTLE time series as differential stretching of line elements along an isochrone source line. To verify this result and to study the effect of different $\delta t$ 's on the recovery of local FTLE time series, we calculate the benchmark backward FTLE fields for the interrogation window with integration time equal to $24 \mathrm{~h}$. Figure $9 \mathrm{a}$ shows an image of the true timevarying FTLE field corresponding to 12:00 UTC 29 September 2010. To give a sense of the changes of the FTLE field during the interrogation window, we may describe the motion of the strong ridges of the field in Fig. 9a toward a northwesterly direction, as shown by the arrow. Figure $9 \mathrm{~b}$ shows the benchmark local FTLE value (black line) at the Kentland Farm during the interrogation window. To generate this plot, we use Eq. (6) and calculate the backward FTLE field every $15 \mathrm{~min}$; then, the time-varying value of FTLE at $(0,0)$ is extracted. Also, to compare the results, the recovered FTLE time series corresponding to $\delta t=0.1 \mathrm{~h}$ is displayed in the same panel by the red line. Figures $8 \mathrm{~b}$ and $9 \mathrm{~b}$ indicate that (i) an optimal $\delta t$ for this example is between 0.1 and $0.5 \mathrm{~h}$, and that (ii) the estimation error is smaller for larger values of the true local FTLE. Therefore, we may observe larger errors of estimation when (true) $\sigma$ is close to zero, e.g., between 00:00 and 04:00 UTC in Fig. 9b. For $\delta t=0.1 \mathrm{~h}$, we observe that the true and approximated local FTLE time series are highly correlated, and their maxima (corresponding to the local maxima of the FTLE field) are also at the same times (within $\delta t= \pm 0.1 \mathrm{~h}$ ). Therefore, with a proper choice of $\delta t \mathrm{~s}$, the recovered local FTLE time series can accurately capture the passage times of moving ridges of a FTLE field. Detecting these ridges is important since they are candidates for hyperbolic LCSs in many geophysical applications (Tallapragada et al., 2011; Haller, 2011; Karrasch, 2012; BozorgMagham et al., 2013).

In addition, we investigate whether we can estimate the distances by using observation II, provided there is necessary information about local velocity and FTLE. Figure 10 is a numerical example that shows that the benchmark distance between the successive source points (black line) is well approximated (red line) by observation II, i.e., Eq. (9). Note 
a)

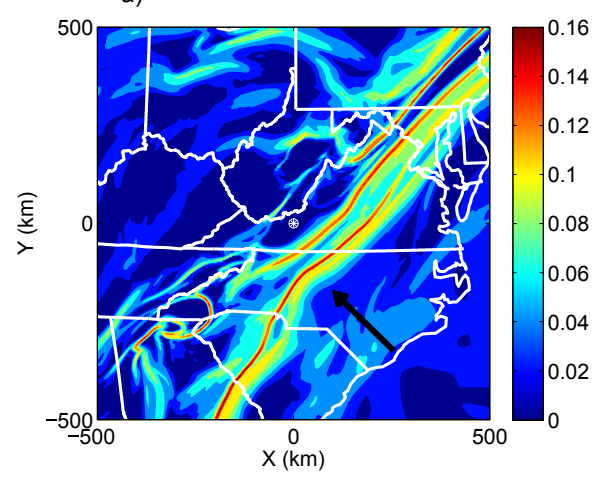

b)

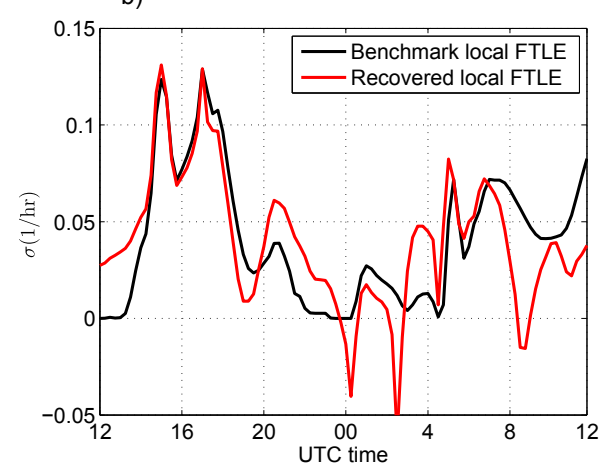

Figure 9. (a) The frozen image corresponding to 12:00 UCT 29 September 2010 of the backward FTLE field during the interrogation window. Integration time is $24 \mathrm{~h}$ for FTLE calculations. The bold arrow shows the general wind direction and the motion of the attracting LCS. (b) The true (black) and recovered (red) local FTLE time series at the reference point $(0,0)$. For the recovered time series (red), $\delta t$ is equal to $0.1 \mathrm{~h}$.

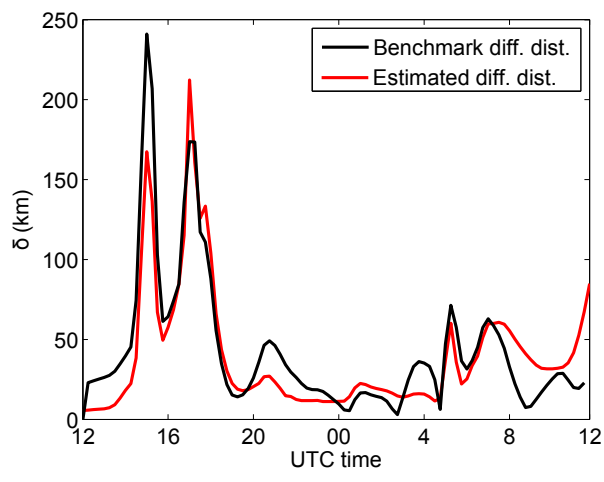

Figure 10. Differential distance between the successive source points on the isochrone source line corresponding to $\delta t=0.25 \mathrm{~h}$. The black line shows the benchmark and the red line shows the approximated time series that is calculated by the local FTLE formula as $\exp \left(|T| \sigma_{\left[t_{1}, t_{2}\right]}^{T}(\boldsymbol{x})\right)\left|\overline{\boldsymbol{v}}\left(\boldsymbol{x}, t_{1}, t_{2}\right) \delta t\right|$. The backward integration time for calculations of the flow maps is $T=24 \mathrm{~h}$ and the interrogation window is 12:00 UTC 29 September to 12:00 UTC 30 September 2010 .

that in this case we have the data of the true local FTLE and the local velocity. In this figure we see that at $\delta t=0.25 \mathrm{~h}$, the estimated differential distance time series is very close to the true answer, and it captures the correct times of the local maxima.

This is an empirically important result, because one can schedule the sampling of geophysical flows (e.g., with drones) based on the available forecast FTLE fields and local velocity such that the successive collected particles originate from the most possible diverse locations. In Fig. 10 it is evident that there are two optimal time intervals, i.e., before and after 16:00 UTC, for maximal diversity monitoring. To interpret this, consider Fig. $7 \mathrm{~b}$ and notice that the geographic extent of the line segment from point 15 to point 16 is much larger than the segment from point 13 to point 14 .
A direct result of the local FTLE observations is the possibility of planning for maximal geographic (and therefore also genetic) diversity monitoring such that the collected particles come from the most separated source locations. This means incorporating greater potential source areas, which could drive a greater diversity of sample collection. Suppose that it is desired to maximize the genetic diversity of microorganisms collected in a sample, assuming that all the collected particles have approximately the same flight time. Results of observation II indicate that to collect samples such that they originate from the most distant locations, one should collect at times corresponding to the maxima of the local FTLE time series (note the high correlation between the distance and the local FTLE time series in Figs. 10 and 9b). To ensure that the particles are coming from significantly separated locations, we may use the topology of the FTLE field and collect the samples on either side of a strong attracting LCS feature that corresponds to a local maximum of $\sigma_{\left[t_{1}, t_{2}\right]}^{T}$, provided there is a short enough time between sampling periods. In this condition, a high value of $\sigma_{\left[t_{1}, t_{2}\right]}^{T}$ as the exponent in Eq. (7) is the reason for having a large $\delta$. Figure 11 schematically shows this strategy when an attracting LCS feature passes over a fixed sampling location, causing a dramatic change in the region of possible source points of collected particles.

As an example in realistic geophysical flow, Fig. 12 shows trajectories of three hypothetical particles that are collected at $(0,-100) \mathrm{km}$ with respect to the reference point. Backward integration time for specifying the corresponding source points, i.e., A, B and C, and the trajectories is $40 \mathrm{~h}$ for those three particles. The sampling times during the interrogation window are 13:40 UTC for the red particle, 14:00 UTC for the blue particle and 14:10 UTC for the green particle. The green and blue particles are collected on one side of an attracting LCS, but the red particle is collected on the other side of the same LCS. As we observe, the source points corresponding to blue and green particles, points $\mathrm{B}$ and $\mathrm{C}$, are close. Meanwhile the source point of the red particle, 


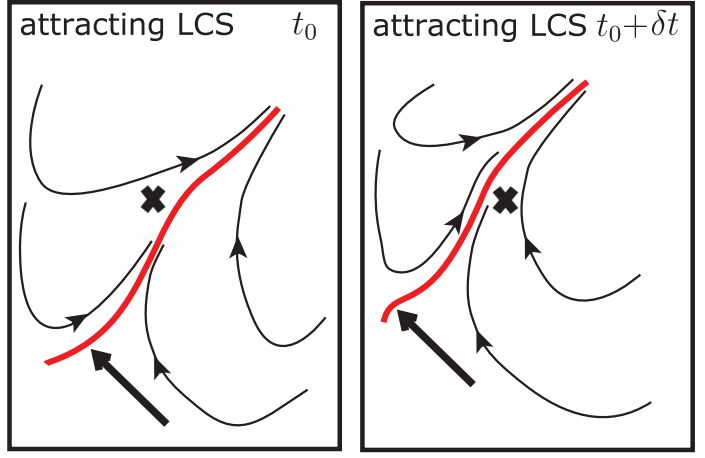

Figure 11. An attracting LCS feature (red) passes over the geographically fixed sampling location (indicated by a bold $\mathbf{x}$ ). Black lines show trajectories of hypothetical particles that are absorbed to a moving attracting LCS. The bold arrow shows the general wind direction and the motion of the attracting LCS at the specified interval. Collected samples on either side of this attracting LCS feature come from two different regions.

point $\mathrm{A}$, is significantly far from the other two particles. An interesting feature of this figure is that the separation of the trajectories does not start from the sampling point, but as is shown, the three trajectories remain close to each other for about $200 \mathrm{~km}$ and then begin to diverge. This observation is directly related to the concept of the FTLE, because $\sigma_{t_{0}}^{T}$ is a function of the "final" separation between nearby particles, and it does not specify the moment of divergence.

Referring to this example, observation II can help us to explain the seeming association of sample diversity with high FTLE. There have been some reports of significant characteristic variation of the collected particles, e.g., genetic types or aerial density of the microbial samples, during short intervals when sampling coincides with a high-value local FTLE, or similarly, passage of a strong LCS over the sampling location (Schmale et al., 2012; Lin et al., 2013). In addition, a direct result of the local FTLE observations is that when the local FTLE value is small during the sampling process, it is expected that the collected particles originate from nearby source points, assuming approximately the same flight times for them. This might be the reason that the characteristics of the microbial samples remain quasi-constant in consecutive collections, but differ as the time between sample collections increases (Lin et al., 2013). This situation is similar to sampling from a coherent set where the FTLE values are generally small (Froyland et al., 2010; Tallapragada and Ross, 2013) and the particles have similar Lagrangian characteristics. Moreover, in cases where we observe significant changes in collected samples while the local FTLE value is small, we speculate that those changes are caused by local sources rather than long-range transport phenomena (Lin et al., 2013). Thus, the local FTLE concept helps us to include or exclude rare/unique microbes from specific source regions. This sets the stage for additional work to be per-

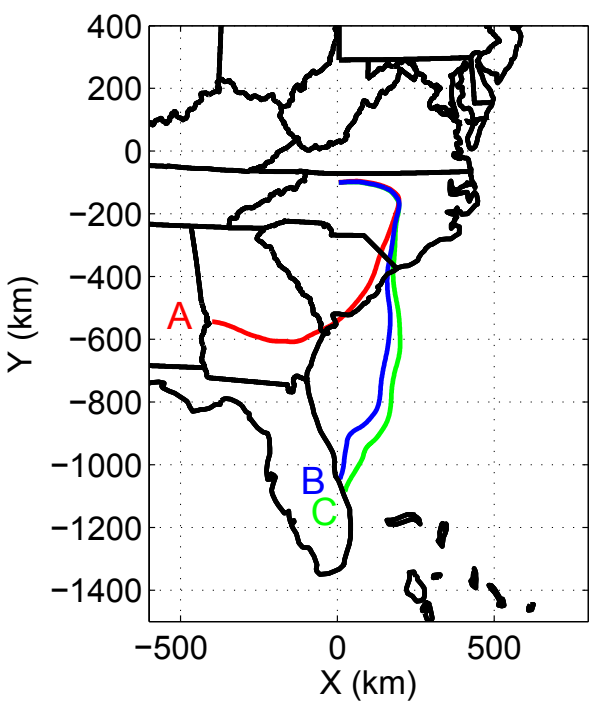

Figure 12. Three calculated trajectories of (hypothetical) collected samples. The red and blue trajectories correspond to the samples on either side of a LCS. The blue and green trajectories correspond to the samples on one side of the same LCS. Sampling times are 13:40, 14:00 and 14:10 UTC during the interrogation window (12:00 UTC 29 September to 12:00 UTC 30 September 2010) for the red, blue and the green particles, respectively. Source points of the collected particles are shown by A, B and C. The integration time for all three particles is $T=40 \mathrm{~h}$.

formed to test hypotheses concerning the presence/absence of the unique microbes at the potential source locations.

\section{Unresolved turbulence and probabilistic regions}

In this section we study the uncertainty in calculation of the source (or destination) points due to unresolved turbulence and also the role of high-value local FTLE and deterministic LCS in separation of the probabilistic source (or destination) regions.

Precise calculation of the source (or destination) point of any collected (or released) particle and the corresponding flow map require high-resolution data of the velocity field. But geophysical data are always discrete and spatially sparse. For example, spatial and temporal resolutions of operational atmospheric data sets vary from the order of 10 to hundreds of kilometers and $3 \mathrm{~h}$ to longer intervals, respectively. Meanwhile, spatiotemporal scales of atmospheric flows can be smaller than the resolution of the available data, and we may lose important Lagrangian phenomena such as turbulent diffusion and small-size eddies if we just consider available data (Csanady, 1973; Rodean, 1996). Therefore, for realistic calculation of the source (or destination) points, it is necessary to consider the uncertainty of the trajectories. For this purpose, we consider a Lagrangian particle dispersion model (LPDM) that provides the stochastic component of 
the velocity with respect to the available deterministic (background) data (Legg and Raupach, 1982; Fay et al., 1995; Draxler and Hess, 1998; Stohl et al., 2005). In LPDM, the velocity vector at each point, $\boldsymbol{v}(\boldsymbol{x}, t)$, is assumed to be the sum of a deterministic term, $\overline{\boldsymbol{v}}(\boldsymbol{x}, t)$, and a random variable, $\boldsymbol{V}(\boldsymbol{x}, \overline{\boldsymbol{v}}, t)$ that depends explicitly on the instantaneous position of the particle $\boldsymbol{x}$, its deterministic velocity $\overline{\boldsymbol{v}}$ at that location and the time $t$; see Eq. (11). Later, we see how this dependency dictates two different solutions for the calculations of the probabilistic source and destination regions (BozorgMagham and Ross, 2015).

$\boldsymbol{v}(\boldsymbol{x}, t)=\overline{\boldsymbol{v}}(\boldsymbol{x}, t)+\boldsymbol{V}(\boldsymbol{x}, \overline{\boldsymbol{v}}, t)$

The stochastic term of Eq. (11) is a Markov-chain process as a function of the velocity deformation tensor and the Lagrangian timescale of the flow field,

$V_{(t+\Delta t)}=R_{\Delta t} V_{t}+\left(1-R_{\Delta t}^{2}\right)^{0.5} \mathcal{N}(0,1) \sqrt{\kappa / T_{\mathrm{L}}}$

where $V$ shows each component of the stochastic velocity term $\boldsymbol{V}$, and the correlation coefficient $R_{\Delta t}$ is a measure of the association between stochastic velocities in successive time steps. Also, $\mathcal{N}$ is a normal distribution with mean zero and unit standard deviation. The correlation coefficient

$R_{\Delta t}=\exp \left(-\Delta t / T_{\mathrm{L}}\right)$

is a function of the integration time step, $\Delta t$, and the Lagrangian timescale of the flow field, $T_{\mathrm{L}}$, which is on the order of $10^{4} \mathrm{~s}$. The term $\kappa$ depends on the gradient of the instantaneous deterministic velocity, $\bar{v}=(\bar{u}, \bar{v})$, the grid size of the meteorological data, $\chi$, and an empirical constant, $c$ :

$\kappa=2^{-0.5}(c \chi)^{2}\left[\left(\frac{\partial \bar{v}}{\partial x}+\frac{\partial \bar{u}}{\partial y}\right)^{2}+\left(\frac{\partial \bar{u}}{\partial x}-\frac{\partial \bar{v}}{\partial y}\right)^{2}\right]^{0.5}$

Because $\kappa$ depends on the gradient of the background velocity, one can easily use the set of Eqs. (11)-(14) for forward integration. Using this set for simple backward integration requires presumption about the position of a particle at specific times, which leads to misleading results. Therefore, we have to consider two distinct cases, (i) calculation of the probabilistic destination region of a released particle, and (ii) calculation of the probabilistic source region of a collected particle. In this study we discuss both cases, but like before, emphasize the probabilistic source regions (corresponding to the backward trajectories). We also revisit the problem of a local FTLE and successive sampling in the presence of unresolved turbulence. Our numerical results show that if successive sampling is performed on either side of a strong attracting LCS (represented by the temporal peaks in the local backward FTLE time series), the probabilistic source regions are significantly separated, similar to the deterministic case.

To focus on the main concerns of this study and to avoid complexity, we proceed with a two-dimensional velocity field similar to the previous sections. However, this approach can be extended to three-dimensional fields by adding an appropriate stochastic term in the extended direction (Rodean, 1996).

\subsection{Probabilistic source and destination regions}

(i) The probabilistic destination region is the probability distribution of the final positions of virtually released particles after integration time $T$ when the initial position is known precisely, e.g., a Dirac delta function. The case of forward integration and related calculations of a probabilistic distribution is equivalent to solving the Fokker-Planck or Kolmogorov forward equations (Rodean, 1996; Risken, 1989), which describe the future of a probability distribution function of a known initial condition that evolves under the dynamics of a system, e.g., a diffusion process.

Because the time-varying vector fields are usually complicated, analytical solutions for probabilistic destination regions are not available, and it is necessary to use numerical solutions. For this aim, we discretize the domain of our interest into sufficiently small boxes and use the Monte Carlo method by releasing a sufficient number of independent particles from a box that includes the release point. Figure 13a shows this procedure. By choosing an appropriate integration time step, we calculate the trajectories. By completion of the integration process, we have a distribution of particles in different boxes. If the total number of released particles is sufficiently large and the boxes' dimensions are sufficiently small, then the ratio of the virtual particles in each box to the total number of released particles shows the probability distribution of the destination region. By increasing the number of virtual particles and decreasing the size of the boxes, the calculated distribution becomes invariant.

(ii) The solution for a probabilistic source region is conceptually the same as solving the Kolmogorov backward problem (Risken, 1989). In mathematical terms, at time $t_{0}-T(T>0$ is the integration time), we investigate for a specific source distribution such that in a future time, i.e., $t_{0}$, the system will be in a given target set. A probabilistic source region cannot be determined by simply performing backward time integration, because $\kappa$ in Eq. (14) and consequently the stochastic velocity term are determined by the instantaneous background velocity that depends on the location and time. Naively applying the backward time integration produces a series of "false" displacement vectors. The cumulative effect of these false displacements yields a false probabilistic source region. To solve this problem, we first discretize the domain of the flow field into small boxes. Then, we shift the starting time to $t_{0}-T$ and consider the velocity field at this new time frame. By this means, we convert this problem into a forward integration problem from $t_{0}-T$ to $t_{0}$. At $t_{0}-T$ we release a sufficient number of independent particles from all boxes of the domain (this step is the major difference between the current and previous case). By forward integration 

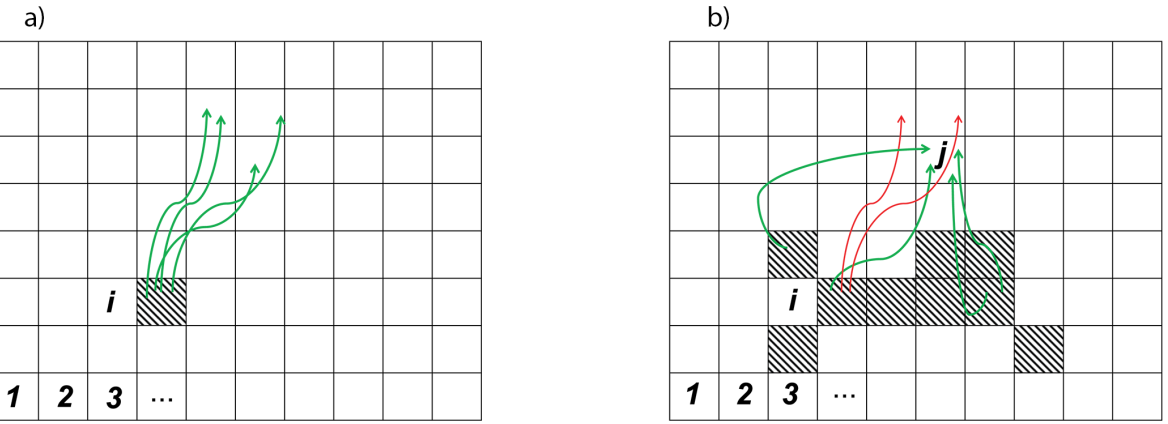

Figure 13. (a) A solution for the probability distribution of a forward case. Virtual particles are released from a box that includes the release location. Distribution of the final positions after integration time $T$ would specify the probabilistic destination region. Calculation of the probabilistic destination region is equivalent to the solution of a Fokker-Planck equation for finding the future probability distribution of an initially known distribution. Trajectories of the released particles from the initial box are shown in green. (b) A solution for the probability distribution of a source region. For a proper forward-time integration, the starting time is shifted to $t_{0}-T$. Virtual particles are released from all the boxes in the domain. Important particles are those that land in the target box, which includes the sampling location. Trajectories of particles that land in the target box are shown by green; other trajectories are shown by red. A solution for the probabilistic source region is conceptually the same as the solution of the backward Kolmogorov equation, where an initial probability distribution is the desired solution such that in a future time the system will have a specified probability distribution.

from $t_{0}-T$ to time $t_{0}$, we find the landing location of all released particles. The influential particles in this procedure are those that land inside the sampling box, e.g., the particles associated with the green trajectories in Fig. 13b. In this figure, those boxes that have contributed to the particles ending up in the target box " $j$ " are hatched. As we observe, there may be particles from contributing boxes that do not land in the target box (shown by red trajectories).

In Fig. 13b the boxes are labeled by $i=1,2, \cdots, n_{\mathrm{b}}$, where $n_{\mathrm{b}}$ is the number of boxes and the sampling box is shown by index $j$. We denote the number of particles that start from box $i$ at time $t_{0}-T$ and are in box $j$ at time $t_{0}$ by $n_{i \rightarrow j}$. We calculate the relative contribution of each source box as

$\gamma_{i}=\frac{n_{i \rightarrow j}}{\sum_{i} n_{i \rightarrow j}}$,

where $\sum_{i} n_{i \rightarrow j}$ shows the total number of particles that land in the sampling (target) box $j$ and $\gamma_{i}$ is the chance of a collected particle coming from a specific box $i$. Therefore, the distribution of $\gamma$ over the domain approximates the probability distribution of the source region. This procedure generates the correct probabilistic source region, but its numerical efficiency is not high because many, e.g., 106 , independent particles are released from all boxes of the domain, but only those particles that land in the sampling box are counted. Thus, there are a huge number of calculated trajectories that are left out. It is not the purpose of this study, but one can increase the efficiency of this procedure by applying some optimization methods, for example, sequential release of particles from large boxes that are inside a circle centered at the sampling box and by identifying the regions with maximum contributions. The radius of that circle can be determined by statistical information about the mean velocity and the inte- gration time. After that, one may focus on those important regions by partitioning them into smaller boxes and increasing the number of released particles to determine fine structures of the probabilistic source region. For more information regarding this problem, one can refer to the STILT project ${ }^{2}$ (Lin et al., 2003; Nehrkorn et al., 2010; Hegarty et al., 2013).

\subsection{Probabilistic source region and local FTLE observations}

To investigate a realistic example of probabilistic source regions and the applicability of the presented observations, we revisit the case study of Sect. 3.2. Figure 14 shows one example of a probabilistic source region where the color intensity determines the relative contribution of each source box. In this case the sampling location is at $(0,-100) \mathrm{km}$ with respect to our reference point. The sampling time is 14:10 UTC 29 September 2010 and the total elapsed time for trajectory calculations is $T=40 \mathrm{~h}$. This figure is the stochastic equivalent of the source point of the particle whose trajectory is shown by the green line in Fig. 12. For this calculation, $10^{5}$ particles are released from each $10 \times 10 \mathrm{~km}$ box. After trial and error experimentation, the search area for this specific problem is considered to be the $900 \mathrm{~km} \times 600 \mathrm{~km}$ rectangular grid shown in Fig. 14b. Considering the size of the boxes, the total number of released particles and calculated trajectories is $5.4 \times 10^{8}$ in each integration time step.

An important point about probabilistic source and destination regions is that although at each time step the stochastic velocity term has a Gaussian distribution (recalling Eq. 12), the final distribution of particles is not necessarily Gaussian. The reason for this fact is the cumulative effects of the vari-

\footnotetext{
${ }^{2}$ http://www.stilt-model.org
} 

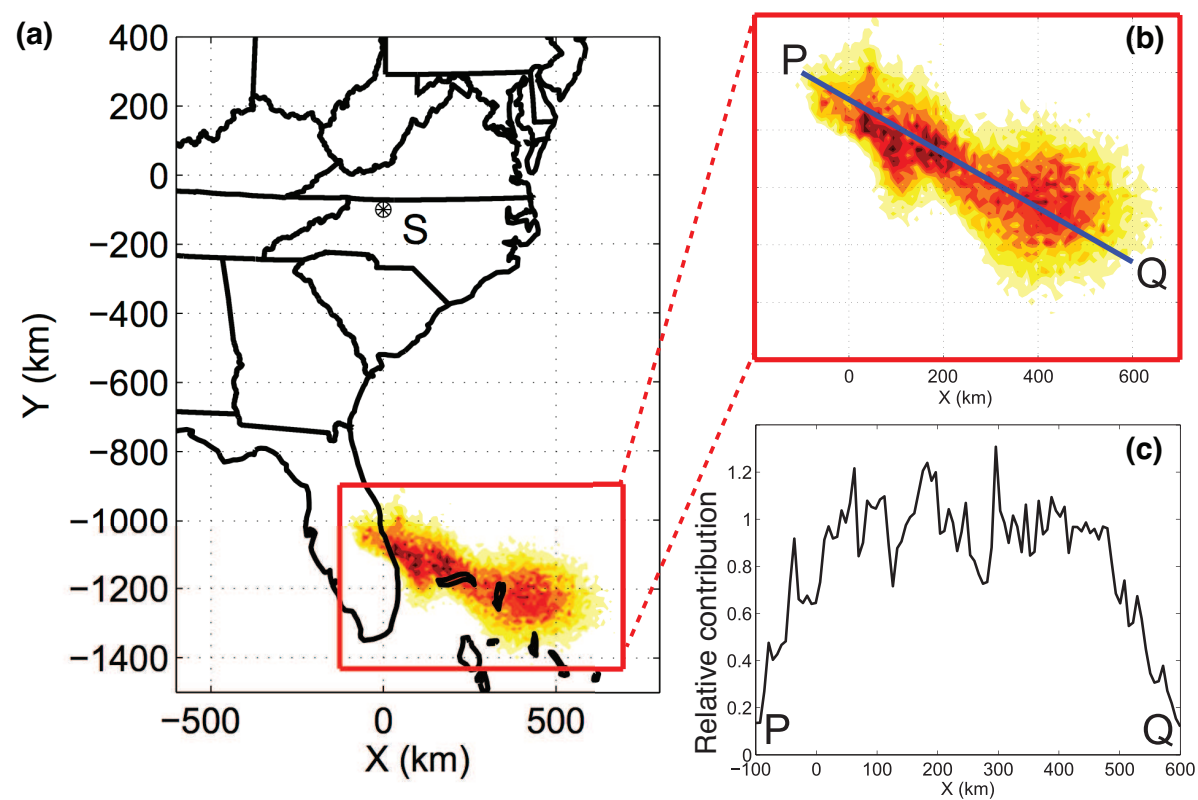

Figure 14. (a) The probabilistic equivalent of the source point of the (virtually) green particle in Fig. 12. The sampling point $S$ is located at $(0,-100) \mathrm{km}$ with respect to our reference point and the sampling time is 14:10 UTC 29 September 2010. (b) Details of the probabilistic source region that is composed of 5400 boxes, each $10 \mathrm{~km} \times 10 \mathrm{~km}$. Color intensity shows the relative contribution of each source box. (c) $\gamma$, the relative contribution of source boxes along the specified line PQ.

ability of the variance of normal distribution, $\sqrt{\kappa / T_{\mathrm{L}}}$, that is, a function of the gradient of instantaneous velocity. In general, for small integration time, the probability distribution of the source (or destination) region is close to a Gaussian distribution, but as the integration time increases, the corresponding distribution diverges from a normal one. For example, visual inspection of Fig. 14b indicates that the final distribution of the probable source points is not Gaussian. In Fig. 14c the relative contribution of the source boxes along the specified line PQ is shown. Standard statistical tests such as the Kolmogorov-Smirnov test (Lilliefors, 1967) confirm that the distribution is not Gaussian.

In Fig. 12 we show that the source locations of two collected particles on either side of an attracting LCS are much further apart than the source points of two successive collected particles on one side of the same LCS. We want to investigate whether this result is still valid in the presence of unresolved turbulence. If that result holds, then in practical applications such as sampling of the microbial structure of the atmosphere, we can have reasonable confidence about the separation of the probabilistic source regions based solely on a deterministic analysis, that is, without performing bothersome probabilistic calculations. Therefore, we study a case where we know its deterministic dynamics. Figure 15 shows the evolution of the probabilistic source regions "A" and "B" (shown in Fig. 15a) corresponding to the (virtually) red and blue particles of Fig. 12, respectively. The total integration time for this example is $40 \mathrm{~h}$. In each panel of this figure we also show the attracting hyperbolic LCSs according to
Haller (2011) and Karrasch (2012). For calculation of each probabilistic region of this figure, $10^{5}$ particles are released from each small $10 \mathrm{~km} \times 10 \mathrm{~km}$ box. By comparing Figs. 12 and $15 \mathrm{a}$, we observe that the probabilistic source regions contain the deterministic source points, and they are significantly separated from each other. Also, we see how the two probabilistic regions contract and become closer to the attracting LCS as they get closer to the sampling point. One noticeable feature in this figure is the difference between the shapes of the two source regions, while the two samplings are separated by only $20 \mathrm{~min}$.

Results of this example show that, similar to the consequences of observation II, the probabilistic source regions corresponding to the collected particles on either side of a deterministic attracting LCS are significantly separated in backward time.

\section{Conclusions}

FTLE fields provide useful information about large-scale transport phenomena and also Lagrangian structures of flow fields, particularly geophysical flows. However, in field experiments the data are on a much more modest scale. Therefore, it is necessary to bridge the gap between the concept of large-scale FTLE fields and local experiments. To fill that gap, we propose a methodology that is an ansatz that is closely related to the concept of local Lyapunov exponents and the direction of maximum expansion in autonomous ordinary differential equation systems (a rigorous mathemati- 

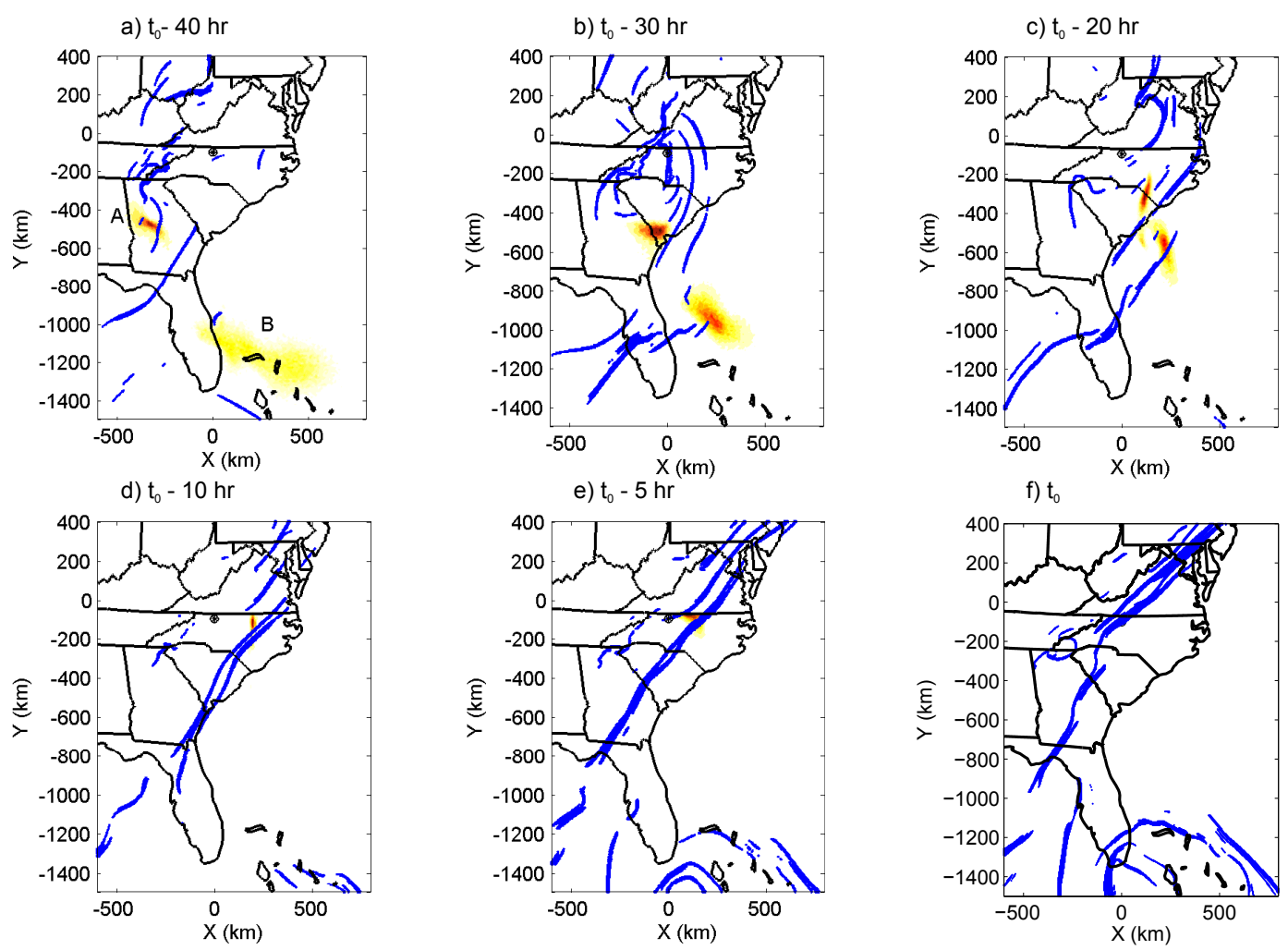

Figure 15. Sequence of the hyperbolic LCSs (blue) and two probabilistic source regions corresponding to two successive samples. Probabilistic regions "A" and "B" (a) correspond to the virtually red and blue particles in Fig. 12. These six panels correspond to 40, 30, 20, 10, 5 and $0 \mathrm{~h}$ before collecting the corresponding samples at 13:40 and 14:00 UTC during the interrogation window (see the Supplement video).

cal formalism for non-autonomous dynamical systems is still needed). Our observations correspond to (i) estimation of the local FTLE, given the local velocity and the distance between sequentially released (or collected) particles, and (ii) estimation of the distances between the destination (or source) points of sequentially released (or collected) particles assuming the availability of the local velocity and local FTLE.

These observations were motivated in part by our previous work examining the dynamics of assemblages of microorganisms in the lower atmosphere. We numerically demonstrate the results of our observations for a periodic velocity field (i.e., a double-gyre), an aperiodic system (i.e., a Rayleigh-Bénard convection model) and real-world wind data. The suggested notion is useful in practical cases where we have samples of particles (e.g., microbes) collected at a fixed location, and we are interested in formulating hypotheses about their origin, structure, and potential transport phenomena driving their atmospheric movement. In addition, we show that the concept of local FTLE and observation II can be applied to scheduling of atmospheric sampling missions to collect high-diversity samples.

We also investigate the unresolved turbulence and the probabilistic description of the source (or destination) points. We use the box discretization method and discuss the important differences between calculation methods of the proba- bilistic source and destination regions. Furthermore, we show that because the stochastic velocity is a function of instantaneous background velocity, the probabilistic source (or destination) regions are not necessarily Gaussian. Finally, we study the probabilistic source regions corresponding to successively collected particles on either side of a strong hyperbolic attracting LCS - or equivalently, a local maximum of the local FTLE time series - and demonstrate that one may trust the estimated results of deterministic calculations of source (or destination) points in realistic geophysical flows.

Results of this study can aid in optimizing the sampling schedules of passive particles and understanding of the outcomes of local observations in geophysical flows, based on large-scale transport features.

\section{The Supplement related to this article is available online at doi:10.5194/npg-22-663-2015-supplement.}

Acknowledgements. This material is based upon work supported by the National Science Foundation under grant numbers CMMI1100263 (Dynamical Mechanisms Influencing the Population Structure of Airborne Pathogens: Theory and Observations) and CMMI-1150456 (Integrating Geometric, Probabilistic, and Topological Methods for Phase Space Transport in Dynamical 
Systems). Part of this research was performed during a visit by S. D. Ross to the Instituto de Ciencias Matemáticas, Madrid, Spain. He thanks ICMAT for its hospitality and support from MINECO ICMAT Severo Ochoa project SEV-2011-0087.

Edited by: V. Lucarini

Reviewed by: C. Harrison and two anonymous referees

\section{References}

Abarbanel, H. D., Brown, R., and Kennel, M. B.: Local Lyapunov Exponents Computed from Observed Data, J. Nonlin. Sci., 2, 343-365, 1992.

Batchelor, G. K.: An Introduction to Fluid Dynamics, Cambridge University Press, 2000.

BozorgMagham, A. E. and Ross, S. D.: Atmospheric Lagrangian Coherent Structures Considering Unresolved Turbulence and Forecast Uncertainty, Commun. Nonlin. Sci. Numer. Simul., 22, 964-979, 2015.

BozorgMagham, A. E., Ross, S. D., and Schmale, D. G.: Realtime Prediction of Atmospheric Lagrangian Coherent Structures Based on Uncertain Forecast Data: An Application and Error Analysis, Physica D, 258, 47-60, 2013.

Branicki, M. and Wiggins, S.: Finite-Time Lagrangian Transport Analysis: Stable and Unstable Manifolds of Hyperbolic Trajectories and Finite-Time Lyapunov Exponents, arXiv preprint arXiv:0908.1129, 2009.

Csanady, G. T.: Turbulent Diffusion in the Environment, vol. 3, Springer, the Netherlands, 1973.

Dellnitz, M., Froyland, G., Horenkamp, C., Padberg-Gehle, K., and Sen Gupta, A.: Seasonal Variability of the Subpolar Gyres in the Southern Ocean: a Numerical Investigation Based on Transfer Operators, Nonlin. Processes Geophys., 16, 655-663, doi:10.5194/npg-16-655-2009, 2009.

Draxler, R. and Hess, G.: An Overview of the HYSPLIT_4 Modelling System for Trajectories, Dispersion and Deposition, Aust. Meteorol. Mag., 47, 295-308, 1998.

Fay, B., Glaab, H., Jacobsen, I., and Schrodin, R.: Evaluation of Eulerian and Lagrangian Atmospheric Transport Models at the Deutscher-Wetterdienst Using ANATEX Surface Tracer Data, Atmos. Environ., 29, 2485-2497, 1995.

Froyland, G., Lloyd, S., and Santitissadeekorn, N.: Coherent Sets for Nonautonomous Dynamical Systems, Physica D, 239, 15271541, 2010.

Haller, G.: A variational Theory of Hyperbolic Lagrangian Coherent Structures, Physica D, 240, 574-598, 2011.

Haller, G.: Lagrangian Coherent Structures, Annual Review of Fluid Mechanics, 47, 137-162, 2015.

Haller, G. and Poje, A.: Finite Time Transport in Aperiodic Flows, Physica D, 119, 352-380, 1998.

Haller, G. and Yuan, G.: Lagrangian Coherent Structures and Mixing in Two-Dimensional Turbulence, Physica D, 147, 352-370, 2000.

Hegarty, J., Draxler, R. R., Stein, A. F., Brioude, J., Mountain, M., Eluszkiewicz, J., Nehrkorn, T., Ngan, F., and Andrews, A.: Evaluation of Lagrangian Particle Dispersion Models with Measurements from Controlled Tracer Releases, J. Appl. Meteorol. Clim., 52, 2623-2637, 2013.
Karrasch, D.: Comment on "A variational Theory of Hyperbolic Lagrangian Coherent Structures, Physica D 240 (2011) 574-598”, Physica D, 241, 1470-1473, 2012.

Kloeden, P. E. and Rasmussen, M.: Nonautonomous Dynamical Systems, Vol. 176, American Mathematical Soc., Providence, Rhode Island, 2011.

Legg, B. and Raupach, M.: Markov-Chain Simulation of Particle Dispersion in Inhomogeneous Flows - the Mean-Drift Velocity Induced by a Gradient in Eulerian Velocity Variance, Bound.Lay. Meteorol., 24, 3-13, 1982.

Lekien, F. and Haller, G.: Unsteady Flow Separation on Slip Boundaries, Phys. Fluids, 20, 097101, doi:10.1063/1.2923193, 2008.

Lilliefors, H. W.: On the Kolmogorov-Smirnov Test for Normality with Mean and Variance Unknown, J. Am. Stat. Assoc., 62, 399402, 1967.

Lin, B., BozorgMagham, A. E., Ross, S. D., and Schmale, D. G.: Small Fluctuations in the Recovery of Fusaria Across Consecutive Sampling Intervals with Unmanned Aircraft $100 \mathrm{~m}$ Above Ground Level, Aerobiologia, 29, 45-54, 2013.

Lin, B., Ross, S. D., Prussin, A. J., and Schmale, D. G.: Seasonal Associations and Atmospheric Transport Distances of Fungi in the Genus Fusarium Collected with Unmanned Aerial Vehicles and Ground-Based Sampling Devices, Atmos. Environ., 94, 385391, 2014.

Lin, J., Gerbig, C., Wofsy, S., Andrews, A., Daube, B., Davis, K., and Grainger, C.: A Near-Field Tool for Simulating the Upstream Influence of Atmospheric Observations: The Stochastic TimeInverted Lagrangian Transport (STILT) Model, J. Geophys. Res.Atmos., 108, doi:10.1029/2002JD003161, 2003.

Mancho, A. M., Small, D., and Wiggins, S.: Computation of Hyperbolic Trajectories and their Stable and Unstable Manifolds for Oceanographic Flows Represented as Data Sets, Nonlin. Processes Geophys., 11, 17-33, doi:10.5194/npg-11-17-2004, 2004.

Mendoza, C. and Mancho, A. M.: Review Article: "The Lagrangian Description of Aperiodic Flows: a Case Study of the Kuroshio Current”, Nonlin. Processes Geophys., 19, 449-472, doi:10.5194/npg-19-449-2012, 2012.

Nehrkorn, T., Eluszkiewicz, J., Wofsy, S. C., Lin, J. C., Gerbig, C., Longo, M., and Freitas, S.: Coupled Weather Research and Forecasting-Stochastic Time-inverted Lagrangian Transport (WRF-STILT) Model, Meteorol. Atmos. Phys., 107, 51-64, 2010 .

Olascoaga, M. J. and Haller, G.: Forecasting Sudden Changes in Environmental Pollution Patterns, P. Natl. Acasd. Sci., 109, 47384743, 2012.

Olascoaga, M. J., Brown, M. G., Beron-Vera, F. J., and Koçak, H.: Brief Communication "Stratospheric Winds, Transport barriers and the 2011 Arctic Ozone Hole", Nonlin. Processes Geophys., 19, 687-692, doi:10.5194/npg-19-687-2012, 2012.

Oseledec, V. I.: A Multiplicative Ergodic Theorem, Lyapunov Characteristic Numbers for Dynamical Systems, Trans. Moscow Math. Soc, 19, 197-231, 1968.

Peng, J. and Peterson, R.: Attracting Structures in Volcanic Ash Transport, Atmos. Environ., 48, 230-239, 2012.

Prussin, A. J., Li, Q., Malla, R., Ross, S. D., and Schmale, D. G.: Monitoring the Long Distance Transport of Fusarium Graminearum from Field-Scale Sources of Inoculum, Plant Disease, 98, 504-511, 2014a. 
Prussin, A. J., Szanyi, N. A., Welling, P. I., Ross, S. D., and Schmale, D. G.: Estimating the Production and Release of Ascospores from a Field-Scale Source of Fusarium Graminearum Inoculum, Plant Disease, 98, 497-503, 2014b.

Prussin, A. J., Marr, L. C., Schmale, D. G., Stoll, R., and Ross, S. D.: Experimental Validation of a Long-Distance Transport Model for Plant Pathogens: Application to Fusarium Graminearum, Agr. Forest Meteorol., 203, 118-130, 2015.

Risken, H.: The Fokker-Planck Equation: Methods of Solution and Applications, in: Springer Series in Synergetics, Vol. 18, Springer-Vlg, 1989.

Rodean, H. C.: Stochastic Lagrangian Models of Turbulent Diffusion, Meteorol. Monogr., 26, 1-84, 1996.

Rosenstein, M. T., Collins, J. J., and De Luca, C. J.: A Practical Method for Calculating Largest Lyapunov Exponents from Small Data Sets, Physica D, 65, 117-134, 1993.

Schmale, D. G. and Ross, S.: Highways in the Sky: Scales of Atmospheric Transport of Plant Pathogens, Annu. Rev. Phytopathol., 53, 591-611, 2015.

Schmale, D. G., Ross, S. D., Fetters, T., Tallapragada, P., WoodJones, A., and Dingus, B.: Isolates of Fusarium Graminearum Collected 40 to 320 Meters Above Ground Level Cause Fusarium Head Blight in Wheat and Produce Trichothecene Mycotoxins, Aerobiologia, 28, 1-11, 2012.

Schmale III, D. G., Dingus, B. R., and Reinholtz, C.: Development and Application of an Autonomous Unmanned Aerial Vehicle for Precise Aerobiological Sampling Above Agricultural Fields, J. Field Robot., 25, 133-147, 2008.
Shadden, S. C., Lekien, F., and Marsden, J. E.: Definition and Properties of Lagrangian Coherent Structures from Finite-Time Lyapunov Exponents in Two-Dimensional Aperiodic Flows, Physica D, 212, 271-304, 2005.

Shadden, S. C., Lekien, F., Paduan, J. D., Chavez, F. P., and Marsden, J. E.: The Correlation Between Surface Drifters and Coherent Structures Based on High-Frequency Radar in Monterey Bay, Deep-Sea Res. Pt. II, 56, 161-172, 2009.

Solomon, T. and Gollub, J. P.: Chaotic Particle Transport in TimeDependent Rayleigh-Benard Convection, Phys. Rev. A, 38, 6280, doi:10.1103/PhysRevA.38.6280, 1988.

Stohl, A., Forster, C., Frank, A., Seibert, P., and Wotawa, G.: Technical note: The Lagrangian Particle Dispersion Model FLEXPART Version 6.2, Atmos. Chem. Phys., 5, 2461-2474, doi:10.5194/acp-5-2461-2005, 2005.

Tallapragada, P. and Ross, S. D.: A Set Oriented Definition of Finite-Time Lyapunov Exponents and Coherent Sets, Commun. Nonlin. Sci. Numer. Simul., 18, 1106-1126, 2013.

Tallapragada, P., Ross, S. D., and Schmale, D. G.: Lagrangian Coherent Structures Are Associated with Fluctuations in Airborne Microbial Populations, Chaos, 21, 033122, doi:10.1063/1.3624930, 2011.

Tanaka, M. L. and Ross, S. D.: Separatrices and Basins of Stability from Time Series Data: An Application to Biodynamics, Nonlin. Dynam., 58, 1-21, 2009. 